\title{
sciendo
}

\section{Do Strike Patterns or Shoe Conditions Have a Predominant Influence on Foot Loading?}

\author{
by \\ Xiaole Sun ${ }^{1}$, Yang Yang, Lin Wang ${ }^{1,2}$, Xini Zhang ${ }^{1}$, Weijie Fu ${ }^{1,2}$
}

\begin{abstract}
This study aimed to explore the effects of strike patterns and shoe conditions on foot loading during running. Twelve male runners were required to run under shoe (SR) and barefoot conditions (BR) with forefoot (FFS) and rearfoot strike patterns (RFS). Kistler force plates and the Medilogic insole plantar pressure system were used to collect kinetic data. SR with RFS significantly reduced the maximum loading rate, whereas SR with FFS significantly increased the maximum push-off force compared to BR. Plantar pressure variables were more influenced by the strike patterns (15 out of 18 variables) than shoe conditions ( 7 out of 18 variables). The peak pressure of midfoot and heel regions was significantly increased in RFS, but appeared in a later time compared to FFS. The influence of strike patterns on running, particularly on plantar pressure characteristics, was more significant than that of shoe conditions. Heel-toe running caused a significant impact force on the heel, whereas wearing cushioned shoes significantly reduced the maximum loading rate. FFS running can prevent the impact caused by RFS. However, peak plantar pressure was centered at the forefoot for a long period, thereby inducing a potential risk of injury in the metatarsus/phalanx.
\end{abstract}

Key words: foot strike patterns, impact force, plantar pressure, shod/barefoot running.

\section{Introduction}

Running is considered to be one of the most important recreational activities (De et al., 2000). It is an increasingly popular activity worldwide due to its high accessibility and low cost. However, the majority of recreational as well as elite runners experience a high incidence of running injury (Fredericson and Misra, 2007). Up to $79 \%$ of endurance runners are injured annually, and $46 \%$ of these injuries are recurrences (Van Gent et al., 2007). Nearly $80 \%$ of running injuries are overuse injuries, which are caused by overloading of the musculoskeletal system of lower extremities (Davis et al., 2017).

Repetitive impact forces experienced during long distance running normally reach the level of magnitude ranging from two to three times the body mass and are considered to induce damage to the musculoskeletal system of the lower extremity (Nordin et al., 2017). In order to reduce the magnitude of impact force and a corresponding high loading rate, the concept of "cushioning" was proposed in shoe manufacturing to reduce such impacts and potential impact-related running injuries. However, no scientific consensus with regard to the effect of cushioned shoes on the impact force and external loading currently exists. Clarke et al. (1983) showed that highly-cushioned shoes did not significantly change the peak impact force during heel-toe running, whereas Shorten and Mientje (2011) found that the peak impact force in cushioned shoes was significantly reduced during running compared with that in standard shoes. During the past 50 years, the incidence of running

\footnotetext{
1 - School of Kinesiology, Shanghai University of Sport, Shanghai 200438, China.

2 - Key Laboratory of Exercise and Health Sciences of Ministry of Education, Shanghai University of Sport, Shanghai 200438,

China.
} 
injuries has not decreased significantly in spite of the emergence of cushion sports shoes (Nigg, 2001). Thus, the role of cushioned shoes in reducing impact force and the rate of running injury remains controversial.

Recently, professional runners and researchers have advocated barefoot running in training, recreation activities, and competitions (Hamill and Gruber, 2017; Lieberman et al., 2010), claiming that it is an effective approach to reduce impact-related injuries (Altman and Davis, 2016; Bonacci et al., 2014). From a biomechanical perspective, one of the clearest differences between barefoot and conventional shod running is the way the foot strikes the ground. Specifically, most barefoot runners use a forefoot strike pattern (Lieberman et al., 2015), whereas approximately $90 \%$ of shod runners land on their heels (Hasegawa et al., 2007). Thus, the foot strike pattern was considered to be a key factor that contributed to the benefit of barefoot running (Shih et al., 2013). The forefoot strike decreases effective mass in the lower extremities, prevents the occurrence of repetitive impact forces during running, and reduces injury rates in runners (Lieberman et al., 2010), particularly patellofemoral pain (Bonacci et al., 2014). However, forefoot runners have higher Achilles tendon loading (Kulmala et al., 2013) and greater ankle joint contact forces (Rooney, 2013) than rearfoot runners. Therefore, the appropriateness for traditionally shod amateur runners to convert to a forefoot strike pattern or barefoot running is questionable. Furthermore, little evidence on the predominant influences of shoe conditions or foot strike patterns on foot loading during running is available. Plantar pressure measurement has been widely used to examine the actual loading applied to the feet (Mei et al., 2015; Queen et al., 2007). Knowledge on how plantar pressure combined with impact forces occur in response to different foot strike patterns and shoe conditions would be useful to better understand variations in foot and lower extremity loading and runningrelated injuries from both movement control and shoe roles.

The purpose of this study was to determine whether strike patterns or shoe conditions had a significant influence on foot loading. Under different strike patterns, ground reaction force, plantar pressure, and impulse during barefoot running (BR) were compared with those during shod running (SR). We hypothesized that 1) SR would significantly reduce the maximum loading rate, and 2) foot strike patterns would have a more pronounced influence than shoe conditions on overall plantar pressure characteristics during running.

\section{Methods}

\section{Participants}

Twelve healthy male recreational runners (age: $21.0 \pm 1.3$ years, body height: $177.1 \pm 4.2 \mathrm{~cm}$, and body mass: $68.1 \pm 7.4 \mathrm{~kg}$ ) volunteered to participate in this study. They all had 3-4 years of running experience and ran with regular RFS. All subjects reported no history of lower extremity injury within the previous 6 months and no vigorous exercise within 24 hours before the experiment. Before the start of the study, participants were familiarized with the experimental protocol and potential risks. Informed written consent was obtained from each participant and approved by the Institutional Review Board of the Shanghai University of Sport.

\section{Instrumentation}

Two $90 \times 60 \times 10 \mathrm{~cm}$ 3D force plates $(9287 \mathrm{~B}$, Kistler Corporation, Switzerland) embedded in the laboratory floor were used to register the ground reaction force (GRF) data at $1200 \mathrm{~Hz}$. An in-shoe plantar pressure measuring system (T\&T Medilogic Medizintechnik GmbH, Schönefeld, Germany) was used to collect plantar pressure data at $120 \mathrm{~Hz}$ during SR and BR with rearfoot and forefoot strike patterns (RFS and FFS, respectively). An insole contained a maximum of 240 force sensors, the number of which depended on insole size and shape, with dimensions of $0.6 \times$ $0.4 \mathrm{~cm}$ and a working dynamic range of $6-640 \mathrm{kPa}$ (Figure 1). Each insole was calibrated using the manufacturer's calibration device prior to the study. A small portable datalogger was attached to the waist of each subject to transfer data to a computer through a wireless connection. The size of the pressure insole was selected according to the shoe code, and the thickness of the insole was $1.2 \mathrm{~mm}$. A timing system with two sets of photocells (Witty-Manual, Microgate, Italy) was used to monitor the running speed. It collected the time elapsed while the participants ran at 3 $\mathrm{m} / \mathrm{s} \pm 5 \%$ along a $20 \mathrm{~m}$ runway (Figure 2 ). The standard running shoes, each with a full-length 
highly-cushioning unit, were used in this study (Figure 3). All the participants adopted the same lacing pattern and wore the same type of running socks to avoid the influence of various shoelaces and socks.

\section{Experimental procedure}

Prior to the test, the participants were required to warm up on a treadmill at a running speed of $2.2 \mathrm{~m} / \mathrm{s}$ for $3 \mathrm{~min}$. They then ran at $3 \mathrm{~m} / \mathrm{s}$ for $1 \mathrm{~min}$ to familiarize themselves with the target speed in the formal test. Subsequently, each performed BR and SR with FFS or RFS at $3 \mathrm{~m} / \mathrm{s} \pm$ $5 \%$ along a $20 \mathrm{~m}$ runway (Figure 4 ). The order of the foot strike patterns and the barefoot/shod condition was randomized. For each participant, three successful trials in which the foot was completely on the force plate were included for each condition.

\section{Data reduction}

GRF data were normalized to body mass (BM). Figure 5 presents a representative vertical GRF (vGRF) time curve during the stance phase of running in different foot strike and shod conditions. The stance phase was identified when vGRF exceeded a threshold of $10 \mathrm{~N}$. The variables of interest included: 1) total contact time (CT); 2) the first peak vGRF (FP), that is, the local maximum vGRF that was commonly obtained from the first $25 \%$ of the stance phase, and the occurrence time $\left.\left(t_{F P}\right) ; 3\right)$ the second peak vGRF (SP), that is, the overall maximum vGRF, and its occurrence time ( $\mathrm{tsP})$; and 4) the peak vertical loading rate (LR) and its occurrence time $\left(t_{L R}\right)$, which was defined as the peak instantaneous slope of the vGRF time curve before the occurrence of FP.

The plantar pressure data were analyzed using Medilogic software (version 4.4). The plantar pressure was calculated dividing the insole area. A regional analysis of each insole was performed by dividing the plantar surface into five regions, namely, forefoot $(40 \%$ of the foot length), midfoot (30\% of the foot length), rearfoot ( $30 \%$ of the foot length), medial $(50 \%$ of the foot width), and lateral (50\% of the foot width). Plantar pressure variables of interest included the following: 1) the peak pressure (PP) of each region and its occurrence time ( $(P P)$; and 2$)$ the pressuretime integral (PTI50), which was defined as the impulse of pressure, and was calculated from 0 to $50 \mathrm{~ms}$ after the foot contact.

\section{Statistics}

All the data were presented as mean \pm standard deviation (SD). A two-way ANOVA with repeated measures was used to examine the effect of foot strike patterns and barefoot or shod conditions on running forces and plantar pressure characteristics (SPSS 17.0, SPSS Inc., Chicago, IL, USA). The intra-class correlation coefficient (ICC) was calculated using absolute agreement in a twoway model. The significance level $\alpha$ was set at 0.05 .

\section{Results}

\section{Ground reaction force}

Overall, the FP and SP were approximately 2 and 2.5 times BM during running with RFS at 3 $\mathrm{m} / \mathrm{s}$, respectively. However, the t $F P$ occurred earlier in the barefoot condition than in the shod condition. SP occurred during running with FFS, but FP did not (Figure 5). A significantly less CT with FFS was observed compared with RFS during both SR and BR (Table 1).

No significant differences in FP were found between BR and SR with RFS. Moreover, t $F P$ occurred earlier during BR than SR with RFS ( $p<$ 0.01 , ICC $>0.5$, Figure 6), whereas LR was higher and $t_{L R}$ occurred earlier during BR compared to during SR $(p<0.01$, ICC $>0.6)$.

The SP was significantly higher in SR than in BR with both RFS and FFS ( $p=0.008$, ICC $>0.7$, Table 1). The $t_{s p}$ occurred later in the shod condition than in the barefoot condition with RFS $(p=0.001$, ICC $>0.6)$ and during BR with FFS compared to RFS ( $p=0.001$, ICC $>0.6)$, but occurred earlier during SR $(p=0.028$, ICC $>0.5)$.

\section{Plantar pressure}

In summary, foot strike patterns ( 15 of the 18 variables) had a more significant effect on the plantar pressure characteristics (Figure 7) than shod conditions (7 of the 18 variables).

First, a significant effect of the foot strike pattern on the PP was found at all plantar regions (Figure 7). Specifically, the PP at the entire foot $(p$ $=0.012$, ICC > 0.7), midfoot $(p<0.001$, ICC $>0.6)$, rearfoot $(p=0.004$, ICC $>0.8)$, and lateral $(p=0.004$, ICC > 0.6) regions was lower in FFS than in RFS in both BR and SR, except for an increased PP at the forefoot $(p=0.002$, ICC $>0.6)$ region in FFS. Additionally, the PP at the medial foot was higher in FFS compared with RFS in SR $(p=0.018$, ICC > $0.6)$, and a significant shoe effect on the PP was 
found at the midfoot $(p=0.013$, ICC $>0.6)$, rearfoot $(p<0.001$, ICC $>0.8)$, and medial regions $(p=0.003$, ICC >0.6). Specifically, the PP at these regions was higher in SR than in BR with RFS. Only the PP at the midfoot region was higher in SR than in BR with FFS ( $p=0.013$, ICC >0.6).

Second, foot strike patterns also had a significant effect on t $p p$ at four plantar regions (Figure 7). Specifically, the tpp at the forefoot $(p<$ 0.001 , ICC $>0.6)$, midfoot $(p=0.038$, ICC $>0.5)$, and rearfoot $(p=0.001$, ICC $>0.6)$ regions occurred later in RFS compared to FFS in SR and BR. However, the $t p p$ at the medial foot $(p<0.001$, ICC > 0.6) region occurred earlier in RFS than in FFS during BR. A significant shoe effect on the tpP was found at the forefoot $(p=0.039$, ICC $>0.6)$ and medial $(p<0.001$, ICC $>0.6)$ foot regions, that is, the $t_{p P}$ at these regions occurred later in SR than in BR with RFS. The tpp at the forefoot occurred later in SR than in BR with FFS ( $p=0.005$, ICC $>0.6)$, whereas the $t_{P p}$ at the medial region occurred earlier $(p=0.038$, ICC > 0.6).

Similar to the PP results, a significant effect of the foot strike pattern on the PTI50 was found at the five plantar regions (Figure 7). Specifically, the PTI50 at the entire foot $(p=0.004$, ICC $>0.6)$, rearfoot $(p=0.013$, ICC $>0.7)$, lateral $(p=0.044$, ICC > 0.6), and medial $(p=0.004$, ICC $>0.6)$ foot regions was lower in FFS than in RFS in both BR and SR, except for a higher PTI 50 at the forefoot $(p$ $<0.001$, ICC > 0.7). Moreover, a significant shoe effect on the PTI50 was found at the forefoot and rearfoot regions. Specifically, the $\mathrm{PTI}_{50}$ at the forefoot region was higher in BR than in SR both with RFS and FFS ( $p=0.011$, ICC > 0.7); however, the $\mathrm{PTI}_{50}$ at the rearfoot region was lower in BR compared to SR $(p<0.001$, ICC $>0.7)$.

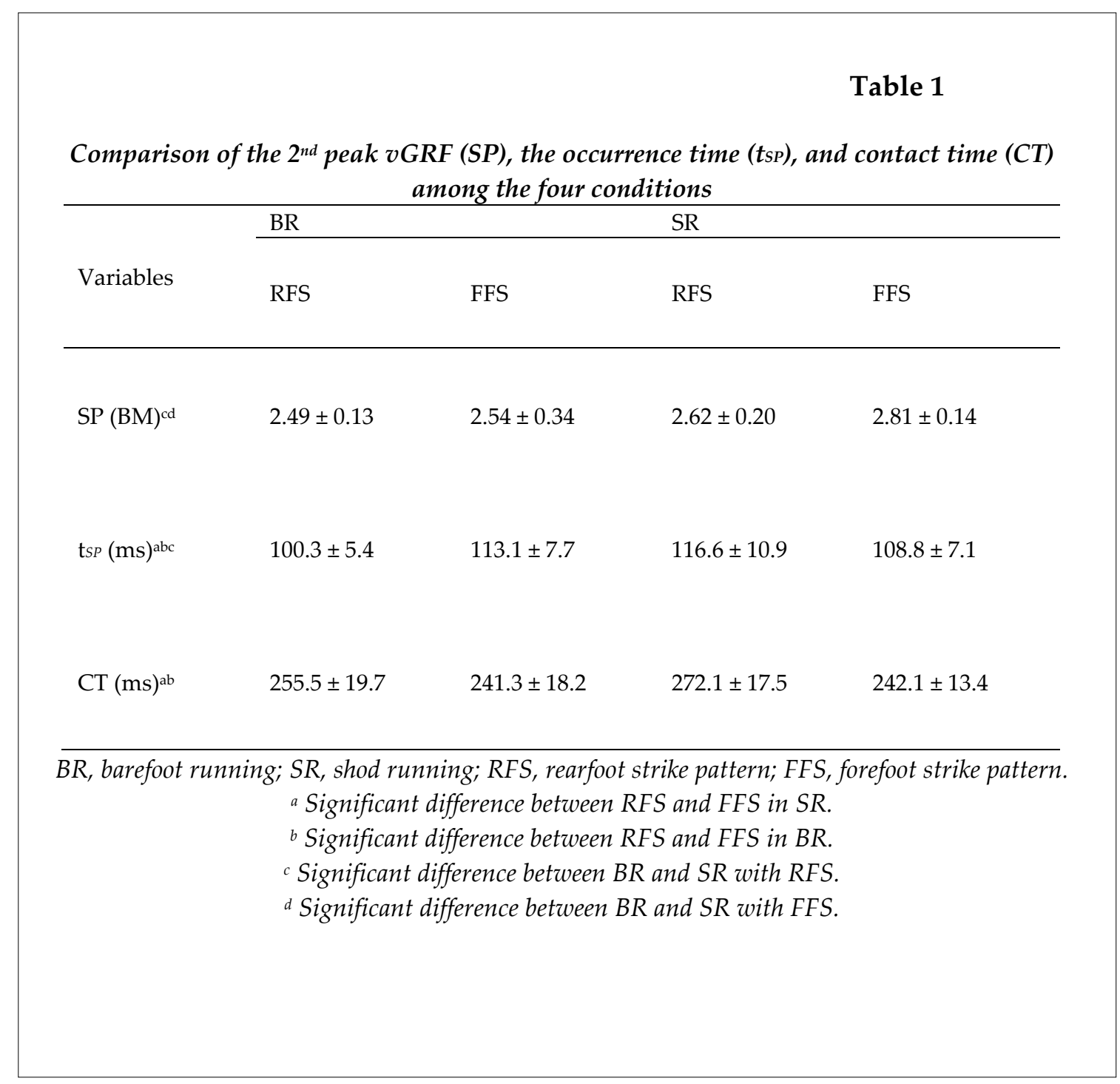



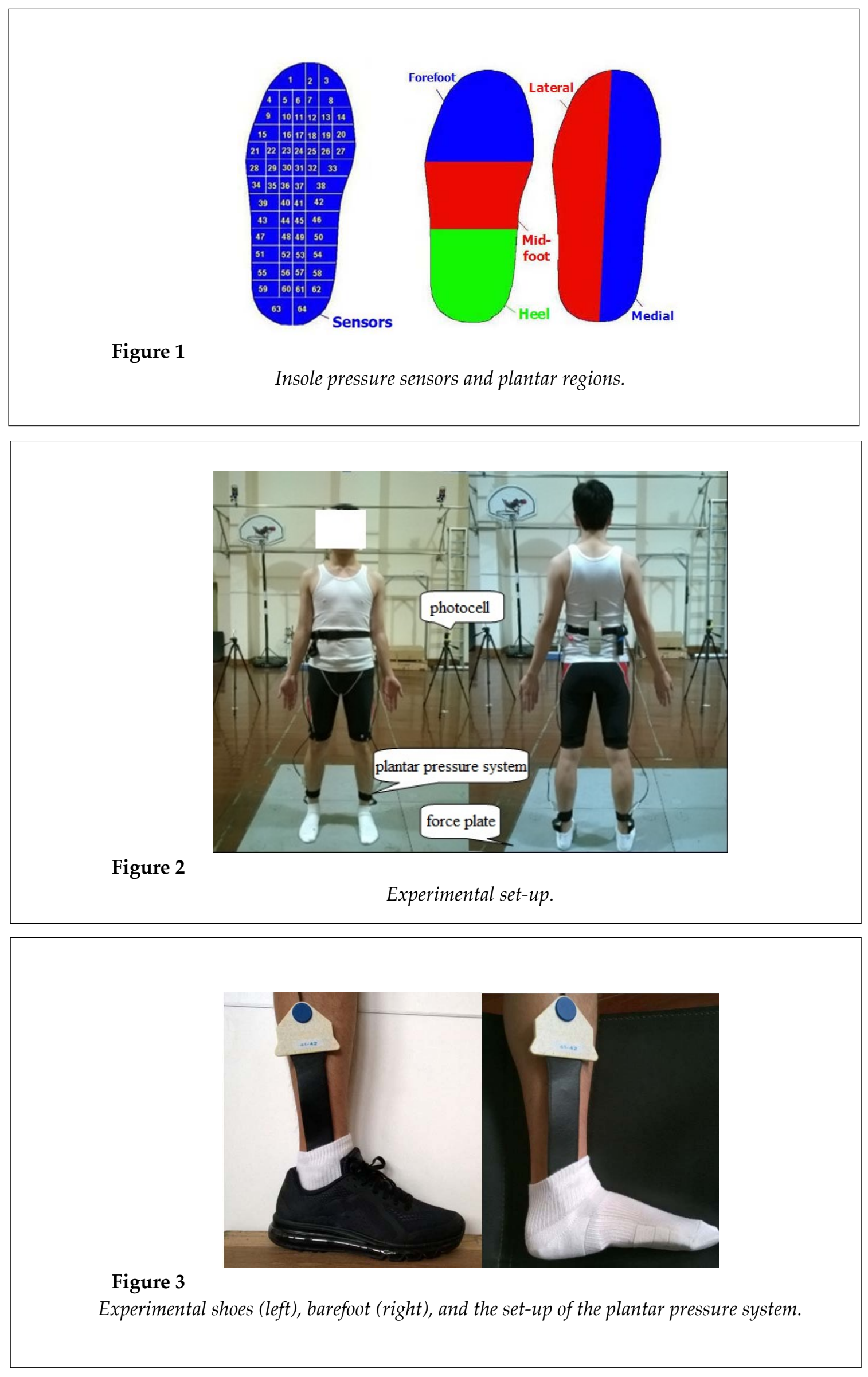


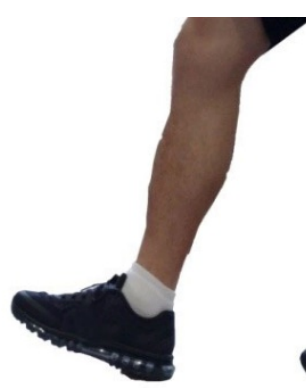

(a)

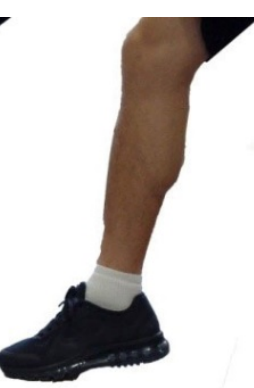

(b)

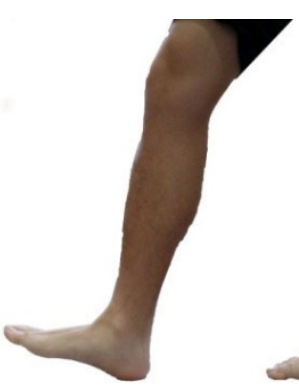

(c)

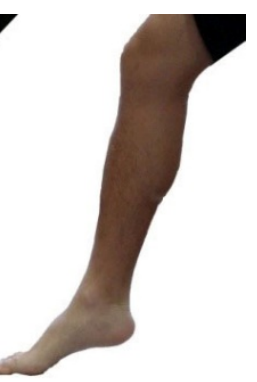

(d)

Figure 4

(a) SR with RFS; (b) SR with FFS; (c) BR with RFS; (d) BR with FFS.
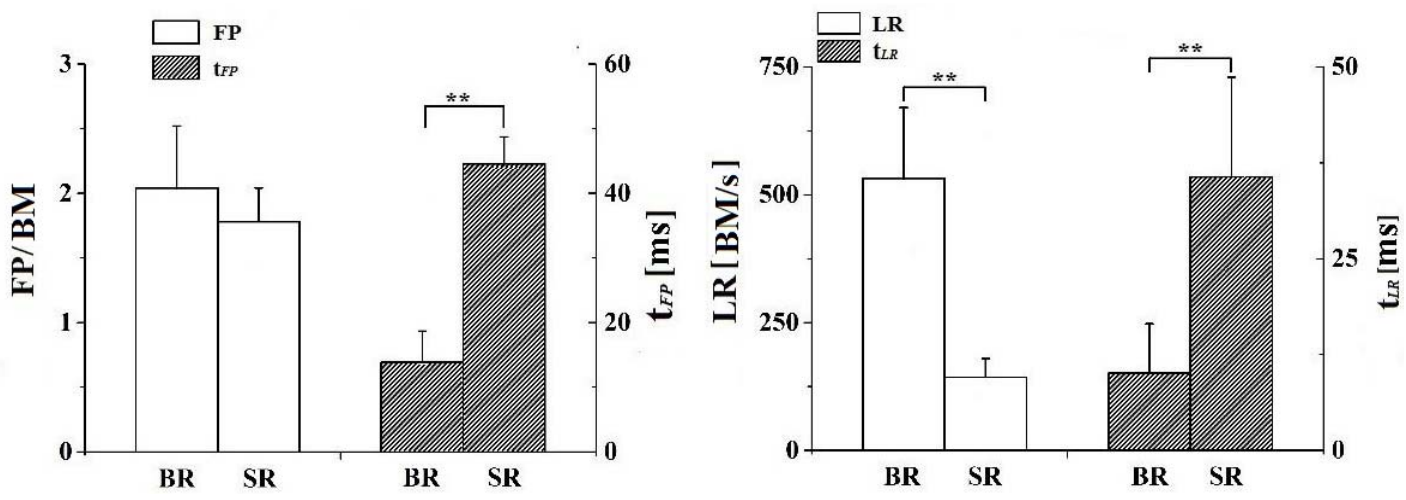

Figure 5

Comparison of the $1^{\text {st }}$ peak $v G R F(F P)$, the corresponding occurrence time ( $\left.t_{F P}\right)$, the peak vertical loading rate $(L R)$ and the occurrence time ( $\left.t_{L R}\right)$ between BR and SR with RFS.
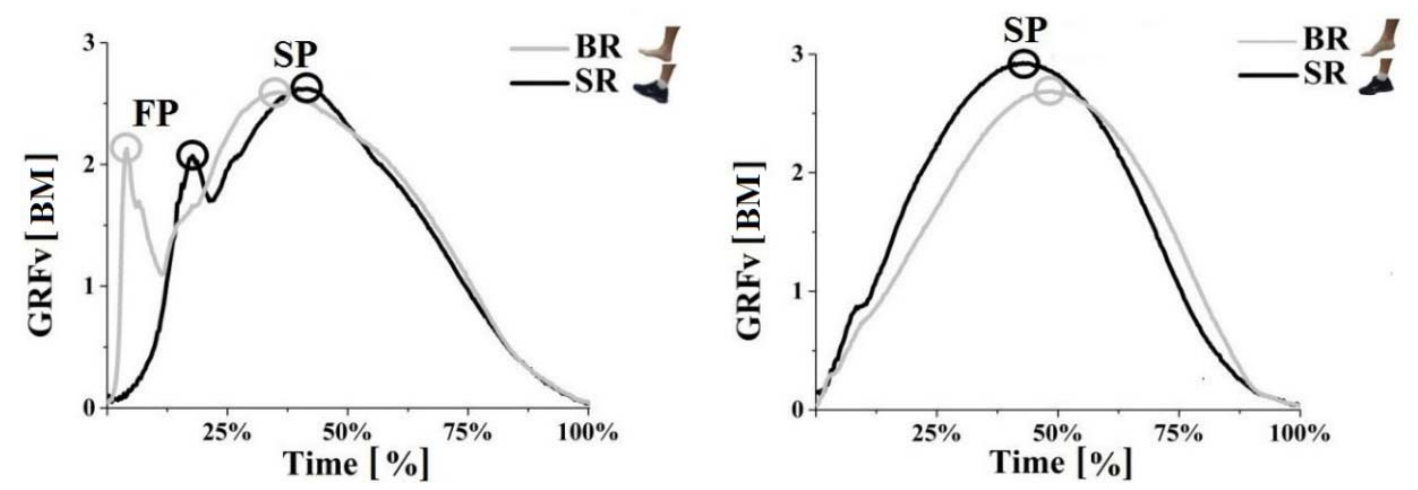

Figure 6

The GRF time curve of barefoot (BR) and shod running (SR) with the rearfoot strike pattern (left) and with forefoot strike pattern (right). 


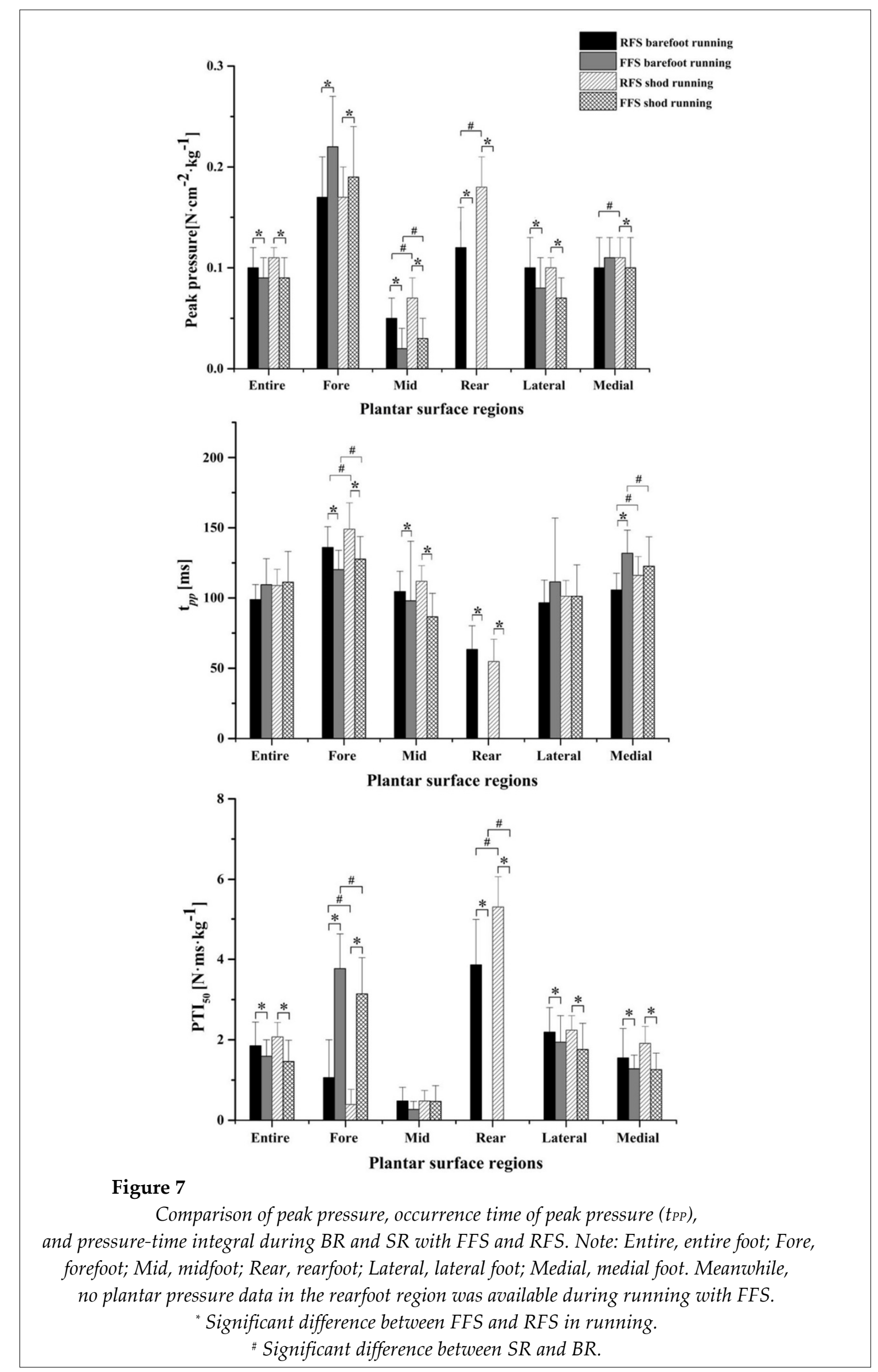




\section{Discussion}

The purpose of this study was to examine whether strike patterns or shoe conditions had a significant influence on foot loading. Our hypothesis was supported by the results which indicated that SR significantly reduced the maximum LR and extended the occurrence of $t_{F P}$ and $t_{L R}$. Moreover, the foot strike patterns (15 out of 18 variables) had a more significant influence than shoe conditions (7 out of 18 variables) on overall plantar pressure characteristics during running.

For the impact forces, no significant difference on FP was observed between BR and $\mathrm{SR}$ at RFS; however, $\mathrm{t}_{F P}$ appeared earlier during BR compared to SR. The lack of significant differences for the FP between shoe conditions supports the results from the majority of impactrelated studies. For instance, De et al. (2000) observed no significant main effect of condition (barefoot vs. shod) for the impact force, which may be because the impact force varies substantially for different effective masses (Lieberman et al., 2010), touchdown speeds (Bruggemann et al., 2011), and lower limb postures (Derrick et al., 2002), but is relatively insensitive to changes in shoe cushioning (Henniget al., 1996). The cushioned shoes were originally designed to attenuate impact force and reduce impact-related injuries (Clarke et al., 1983). Our results, together with those previously mentioned, indicate that cushioned running shoes do not significantly change the FP. On the other hand, LR and $t_{L R}$ were higher and occurred earlier during BR than SR. Similarly, the external loading rate was significantly higher in BR than in SR (De et al., 1994; Komi et al., 1987; Lees, 1988). More compliant shoe cushioning has been found to delay the time at which the $t_{F P}$ occurs and hence reduced the overall loading rate, whereas the magnitude of the impact force was unaffected (Heidenfelder et al., 2010; Wit et al., 2010). In addition, a meta-analysis reported a significant relationship between vertical load rates and tibial stress fractures in RFS runners (Zadpoor and Nikooyan, 2011). Our results suggest that SR does not change peak impacts, yet reduces the LR and extends the occurrence of $t_{L R}$, indicating that RFS is not suitable for BR, whereas cushioned shoes can be beneficial in reducing the LR for runners with RFS.
For the FFS, no FP occurred (Figure 5). Thus, switching the foot strike to FFS may prevent vertical peak impact and decrease the peak loading rate, reducing the risk for impact-related injuries compared with RFS (Boyer et al., 2014; Cheung and Davis, 2011; Crowell and Davis, 2011; Giandolini et al., 2013; Lieberman et al., 2010). Some studies reported the benefits of using FFS. Daoud et al. (2012) reported that in a collegiate cross country team, RFS runners sustained medically diagnosed repetitive stress injuries twice as often as FFS runners. Moreover, transitioning from RFS to FFS prevents running injuries. Diebal et al. (2012) investigated a group of U.S. military cadets who presented anterior compartment syndrome and high intracompartmental pressures and who underwent transition to FFS. All the subjects demonstrated significant reduction in their intracompartmental pressure and were able to complete a $5 \mathrm{~km}$ run without pain.

On the other hand, our findings showed the SP was significantly larger in SR than in BR with FFS. A possible explanation is that the cushioning attribute in the forefoot of shoes enables forefoot landing with a flat placement and stimulates the foot to push off the ground rapidly and heavily (Keijsers et al., 2013; Shorten, 2011). Meanwhile, a significantly less CT occurred with FFS compared with RFS during SR and BR. These results indicate that SR with FFS can prevent peak impact, that is, FP, and produce large active forces, that is, SP, which may reduce the incidence of running injuries and improve performance.

The PP, its occurrence time ( $(t P)$, and PTI 50 were also recorded for the analysis of foot loading characteristics. Based on the results, we found that foot strike patterns had a more significant effect on plantar pressure characteristics compared with shod conditions. In particular, of the 18 variables, 15 were significantly affected by the foot strike pattern, and seven were significantly affected by shoe conditions.

During RFS running, the pressure center moved from the rearfoot to the forefoot, whereas during FFS running, the pressure was concentrated in the forefoot region (Figure 7). Generally, plantar pressure provides information on the distribution of force, and this information can be related to potential damaging effects to local tissues (Rosenbaum and Becker, 2010). In the 
current study, we found that the PP in the forefoot was the greatest (Figure 7). The higher forefoot loads may be relevant to metatarsal stress fractures (Hockenbury, 1999). With regard to the effect of foot strike patterns on regional pressure in our study, significant reductions in PP and PTI50 were observed in the entire foot, rearfoot, and lateral regions in FFS in both BR and SR, except for an increased PP at the forefoot region in FFS. Similar findings were reported by Kernozek et al. (2014), who showed that PP and PTI at heel and midfoot regions were higher in RFS than in FFS during running when a minimalist footwear was used, but were lower in PP and PTI at the forefoot. Furthermore, Warne et al. (2013) found that the increase in plantar pressure in the forefoot region during running altered the foot strike pattern from RFS to FFS with barefootsimulated minimalistic shoes on a treadmill. Changes in plantar pressure can provide accurate data on how the foot is loaded with respect to the supporting surface, given that unnatural or localized loading may predict or indicate injury risk (Orlin and Mcpoil, 2000), particularly tibial and metatarsal stress fractures (Davis et al., 2011). The reduction in plantar pressure during running may represent a possibility for injury reduction as impact and pressure have been linked to runningrelated injury (Davis et al., 2004; Macdermid et al., 2017). Collectively, our findings indicate that FFS may reduce the risk of impact-related injuries in contrast to RFS. However, transitioning from RFS to FFS acutely may also increase forefoot loading.

Regarding the shoe effect on regional pressure with RFS, the PPs at midfoot, rearfoot, and medial regions were significantly reduced in BR compared to SR. The PP at the midfoot region was lower in BR than in SR with FFS, and the $\mathrm{PTI}_{50}$ at the forefoot region was higher in BR than in SR with both RFS and FFS. However, the PTI 50 at the rearfoot region was lower in BR than in SR. These findings are partially supported by the study of Bergstra et al. (2015), who showed that the PP and PTI of the forefoot region increased with the minimalist shoes in contrast to those in standard shoes. The PP and PTI in the heel region did not differ between the two shoe types. In the midfoot region, PP was reduced in minimalist running shoes. The difference between our results and those of Bergstra et al. (2015) may be attributed to differences in gender, testing shoes, and running speed.

The key outcome of this study was that the average PP and average PTI50 of the entire foot were significantly reduced with FFS compared to RFS. Moreover, the lowest PP and PTI50 were obtained during SR with FFS. Combined with the results of the impact and maximum vertical GRF, SR with FFS may prevent impact and improve performance. The current findings indicate that FFS may be the main factor that contributes to the benefit of BR. Meanwhile, the pressure concentrated at the forefoot for a long time with FFS increases the risk of overuse injury at the metatarsal area (Shakoor and Block, 2006). Therefore, a suitable pair of running shoes is needed for runners with either FFS or RFS to reduce plantar pressure during running.

The limitations of the current study are acknowledged along with proposed future directions for research. First, we only considered the acute effects of shoe and foot strike pattern condition on running impacts. Therefore, the assessment of lower limb kinematics, accompanied with joint kinetics and muscle forces or activation, is warranted to provide further evidence of neuro-musculoskeletal reactions. Second, a long-term effect of the shoe and foot strike pattern should be considered. Highlighting the effect of gender is highly recommended.

\section{Conclusion}

The influence of strike patterns on running is more significant than shoe conditions, which was observed in plantar pressure characteristics. Heel-toe running caused a significant impact force on the heel, but cushioned shoes significantly reduced the maximum loading rate. Meanwhile, although forefoot running can prevent impact, peak plantar pressure was centered at the forefoot for a long period, inducing a potential risk of injury in the metatarsus/phalanx. Plantar pressure on the forefoot with RFS was lesser and push-off force was greater when cushioned shoes were used than when running barefoot. 


\section{Acknowledgements}

The authors would like to acknowledge supports for the study from the National Natural Science Foundation of China (11772201, 11572202, 11572213) and the Innovation Program of Shanghai Municipal Education Commission (14YZ125).

\section{References}

Altman AR, Davis IS. Prospective comparison of running injuries between shod and barefoot runners. $\mathrm{Br} \mathrm{J}$ Sports Med, 2016; 50: 476-480

Bergstra SA, Kluitenberg B, Dekker R, Bredeweg SW, Postema K. Running with a minimalist shoe increases plantar pressure in the forefoot region of healthy female runners. J Sci Med Sport, 2015; 18: 463-468

Bonacci J, Vicenzino B, Spratford W, Collins W. Take your shoes off to reduce patellofemoral joint stress during running. Br J Sports Med, 2014; 48: 425-428

Boyer ER, Rooney BD, Derrick TR. Rearfoot and midfoot or forefoot impacts in habitually shod runners. Med Sci Sports Exerc, 2014; 46: 1384-1391

Brüggemann GP, Brüggemann L, Heinrich K, Mller M, Niehoff A. Biological tissue response to impact like mechanical loading. Footwear Science, 2011; 3: 13-22

Cheung RT, Davis IS. Landing pattern modification to improve patellofemoral pain in runners: a case series. J Orthop Sports Phys Ther, 2011; 41: 914-919

Clarke TE, Frederick EC, Cooper LB. Effects of shoe cushioning upon ground reaction forces in running. Int J Sports Med, 1983; 4: 247-251

Crowell HP, Davis IS. Gait retraining to reduce lower extremity loading in runners. Clin Biomech, 2011; 26: $78-83$

Daoud AI, Geissler GJ, Wang F, Saretsky J, Daoud YA, Lieberman DE. Foot strike and injury rates in endurance runners: a retrospective study. Med Sci Sports Exerc, 2012; 44: 1325-1334

Davis I, Milner CE, Hamill J. Does increased loading during running lead to tibial stress fractures? Med Sci Sports Exerc, 2004; 36: 58

De CD, Aerts P, Kunnen M. The mechanical characteristics of the human heel pad during foot strike in running: an in vivo cineradiographic study. J Biomech, 1994; 27: 1213-1222

De WB, De WB, Aerts P. Biomechanical analysis of the stance phase during barefoot and shod running. $J$ Biomech, 2000; 33: 269-278

Derrick TR, Dereu D, Mclean SP. Impacts and kinematic adjustments during an exhaustive run. Med Sci Sports Exerc, 2002; 34: 998-1002

Diebal AR, Gregory R, Alitz C, Gerber JP. Forefoot running improves pain and disability associated with chronic exertional compartment syndrome. Am J Sports Med, 2012; 40: 1060-1067

Fredericson M, Misra AK. Epidemiology and aetiology of marathon running injuries. Sports Med, 2007; 37: 437-439

Giandolini ME, Horvais N, Farges Y, Samozino P, Morin JB. Impact reduction through long-term intervention in recreational runners: midfoot strike pattern versus low-drop/low-heel height footwear. Eur J Appl Physiol, 2013; 113: 2077-2090

Giuliani J, Masini B, Alitz C, Owens BD. Barefoot-simulating footwear associated with metatarsal stress injury in 2 runners. Orthopedics, 2011; 34: 320-323

Hamill J, Gruber AH. Is changing footstrike pattern beneficial to runners? J Sport Health Sci, 2017; 6: 146-153

Hasegawa H, Yamauchi T, Kraemer WJ. Foot strike patterns of runners at the 15-km point during an elitelevel half marathon. J Strength Cond Res, 2007; 21: 888-893

Heidenfelder J, Sterzing T, Milani TL. Systematically modified crash-pad reduces impact shock in running shoes. Footwear Science, 2010; 2: 85-91

Hennig EM, Valiant GA, Liu Q. Biomechanical variables and the perception of cushioning for running in various types of footwear. J Appl Biomech, 1996; 12: 143-150

Hockenbury RT. Forefoot problems in athletes. Med Sci Sports Exerc, 1999; 31: 448-458

Keijsers NL, Stolwijk NM, Louwerens JW, Duysens J. Classification of forefoot pain based on plantar pressure measurements. Clin Biomech, 2013; 28: 350-356 
Kernozek TW, Meardon S, Vannatta CN. In-shoe loading in rearfoot and non-rearfoot strikers during running using minimalist footwear. Int J Sports Med, 2014; 35: 1112-1117

Komi PV, Gollhofer A, Schmidtbleicher D, Frick U. Interaction between man and shoe in running: considerations for a more comprehensive measurement approach. Int J Sports Med, 1987; 8: 196-202

Kulmala JP, Avela J, Pasanen K, Parkkari J. Forefoot strikers exhibit lower running-induced knee loading than rearfoot strikers. Med Sci Sports Exerc, 2013; 45: 2306-2313

Lees A. The role of athlete response tests in the biomechanical evaluation of running shoes. Ergonomics, 1988; 31: 1673-1681

Lieberman DE, Castillo ER, Otarolacastillo E, Sang MK, Sigei TK. Variation in foot strike patterns among habitually barefoot and shod runners in Kenya. Plos One, 2015; 10: e0131354

Lieberman DE, Venkadesan M, Werbel WA, Daoud AI, Andrea SD, Davis IS. Foot strike patterns and collision forces in habitually barefoot versus shod runners. Nature, 2010; 463: 531-535

Macdermid PW, Fink PW, Stannard SR. Shock attenuation, spatio-temporal and physiological parameter comparisons between land treadmill and water treadmill running. J Sport Health Sci, 2017; 6: 482-488

Mei Q, Fernandez J, Fu W, Feng N, Gu Y. A comparative biomechanical analysis of habitually unshod and shod runners based on a foot morphological difference. Hum Mov Sci, 2015; 42: 38-53

Nigg BM. The role of impact forces and foot pronation: a new paradigm. Clin J Sport Med, 2001; 11: 2-9

Nordin AD, Dufek JS, Mercer JA. Three-dimensional impact kinetics with foot-strike manipulations during running. J Sport Health Sci, 2017; 6: 489-497

Orlin MN, Mcpoil TG. Plantar pressure assessment. Phys Ther, 2000; 80(4): 399-409

Queen RM, Haynes BB, Hardaker WM, Garrett WE. Forefoot loading during 3 athletic tasks. Am J Sports Med, 2007; 35: 630-636

Rooney BD. Joint contact loading in forefoot and rearfoot strike patterns during running. J Biomech, 2013; 46: 2201-2206

Rosenbaum D, Becker HP. Plantar pressure distribution measurements. Foot Ankle Surg, 2010; 3: 1-14

Shakoor N, Block JA. Walking barefoot decreases loading on the lower extremity joints in knee osteoarthritis. Arthritis Rheumatol, 2006; 54: 2923-2927

Shih Y, Lin KL, Shiang TY. Is the foot striking pattern more important than barefoot or shod conditions in running? Gait Posture, 2013; 38: 490-494

Shorten M, Mientje MI. The heel impact force peak during running is neither heel nor impact and does not quantify shoe cushioning effects. Footwear Science, 2011; 3: 41-58

Shorten MR. Biomechanics of sports shoes, by Benno M. Nigg. Footwear Science, 2011; 3: 1-2

Van Gent RN, Siem D, Van MM, Van Os AG, Biermazeinstra SM. Incidence and determinants of lower extremity running injuries in long distance runners: a systematic review. Br J Sports Med, 2007; 41: 469480

Davis IS, Rice HM, Wearing SC. Why forefoot striking in minimal shoes might positively change the course of running injuries. J Sport Health Sci, 2017; 6: 154-161

Warne JP, Kilduff SM, Gregan BC, Nevill AM, Moran KA, Warrington GD. A 4-week instructed minimalist running transition and gait-retraining changes plantar pressure and force. Scand J Med Sci Sports, 2013; 24: $964-973$

Wit BD, Clercq DD, Lenoir M. The effect of varying midsole hardness on impact forces and foot motion during foot contact in running. J Appl Biomech, 2010; 11: 395-406

Zadpoor AA, Nikooyan AA. The relationship between lower-extremity stress fractures and the ground reaction force: a systematic review. Clin Biomech, 2011; 26: 23-28

\section{Corresponding author:}

\section{Weijie Fu, PhD.}

School of Kinesiology, Shanghai University of Sport;

200438 Shanghai, China

Email: fuweijie315@163.com; fuweijie@sus.edu.cn 